\title{
Isometric multiplication operators and weighted composition operators of BMOA
}

\section{Ligang Geng ${ }^{1 *}$ (1)}

\section{"Correspondence:}

glgtju@hotmail.com

'School of Mathematics and

Statistics, Chongqing Technology

and Business University, 19 Xuefu

Avenue, Nan'an District, Chongqing, China

\begin{abstract}
Let $u$ be an analytic function in the unit disk $\mathbb{D}$ and $\varphi$ be an analytic self-map of $\mathbb{D}$. We give characterizations of the symbols $u$ and $\varphi$ for which the multiplication operator $M_{u}$ and the weighted composition operator $M_{u, \varphi}$ are isometries of BMOA.
\end{abstract}

MSC: 46B04; 46E15; 47B33; 47B38

Keywords: Isometric; Multiplication Operator; Weighted Composition Operator; BMOA

\section{Introduction}

In order to state our results, we first introduce some notations and definitions. Let $\mathbb{D}$ denote the open unit disk in the complex plane $\mathbb{C}$, that is, $\mathbb{D}=\{z:|z|<1, z \in \mathbb{C}\}$. Its boundary is the unit circle $\partial \mathbb{D}=\{z:|z|=1, z \in \mathbb{C}\}$. A lot of analytic function spaces over $\mathbb{D}$ have been introduced in last half-century, such as Hardy, Bergman, Besov, Bloch, and Dirichlet spaces.

Let $H^{2}$ denote the classical analytic Hardy space on the unit disk $\mathbb{D}$. We will consider the multiplication operator $M_{u}: f \rightarrow u f$ of BMOA space, which consists analytic functions $f \in$ $H^{2}$ whose boundary functions have bounded mean oscillation on $\partial \mathbb{D}$. In this manuscript we suppose that $u$ is analytic in $\mathbb{D}$ and continuous on $\partial \mathbb{D}$. The norm of the BMOA space is defined as

$$
\|f\|_{\mathrm{BMOA}}=|f(0)|+\|f\|_{*},
$$

where

$$
\|f\|_{*}=\sup _{a \in \mathbb{D}}\left\|f\left(\sigma_{a}\right)-f(a)\right\|_{2} .
$$

Here $\|\cdot\|_{2}$ is the norm of $H^{2}(\mathbb{D})$ and $\sigma_{a}$ is the conformal automorphism of $\mathbb{D}, \sigma_{a}(z)=\frac{a-z}{1-\bar{a} z}$ for $a, z \in \mathbb{D}$. There are some equivalent norms of the BMOA space. For example, for a measurable complex-valued function $f$ defined on $\partial \mathbb{D}$, define

$$
f_{I}=\frac{1}{|I|} \int_{I} f\left(e^{i t}\right) d t
$$

(c) The Author(s) 2021. This article is licensed under a Creative Commons Attribution 4.0 International License, which permits use, sharing, adaptation, distribution and reproduction in any medium or format, as long as you give appropriate credit to the original author(s) and the source, provide a link to the Creative Commons licence, and indicate if changes were made. The images or other third party material in this article are included in the article's Creative Commons licence, unless indicated otherwise in a credit line to the material. If material is not included in the article's Creative Commons licence and your intended use is not permitted by statutory regulation or exceeds the permitted use, you will need to obtain permission directly from the copyright holder. To view a copy of this licence, visit http://creativecommons.org/licenses/by/4.0/. 
where $I \subset \partial \mathbb{D}$ is an arc and $|I|$ is the length of $I$, normalized so that $|I| \leq 1$. The function $f$ has bounded mean oscillation if the seminorm

$$
\|f\|_{*}^{\prime}=\sup _{I} \frac{1}{|I|} \int_{I}\left|f\left(e^{i t}\right)-f_{I}\right| d t<\infty
$$

as $I$ ranges over all $\operatorname{arcs}$ in $\partial \mathbb{D}$, then BMOA $=\left\{f \in H^{1}:\|f\|_{*}^{\prime}<\infty\right\}$, this can be seen in [1-3].

Properties of multiplication operators have been studied on various classical Banach spaces of analytic functions in the unit disk $\mathbb{D}$, such as Hardy, Bergman, and Bloch spaces. In [4], the author studied the invertible and Fredholm multiplication operators on Bergman spaces. In [5], the authors studied the compact multiplication operators with BMO symbols. In [6], the authors studied the $C^{*}$-algebra in the commutant of the multiplication operators with the symbol on the finite-dimensional Bergman spaces. In [7], the authors studied the commutants and reducing subspaces of multiplication operators on the Bergman spaces.

Properties of linear isometries of those spaces have received a great deal of attention. Readers can see parts of research in this direction in [8-13]. An interesting fact is that the linear isometries of spaces of continuous functions and some spaces of analytic functions, such as Hardy and Bergman spaces, in the unit disk $\mathbb{D}$ have the forms of weighted composition operators. There are some studies of the properties of isometric and weighted composition operators on spaces of analytic functions on $\mathbb{D}$ and other domains; these can be seen in [14-17]. In [18], the authors studied isometric pointwise multipliers of weighted Bergman, Bloch, and Dirichlet-type spaces in the unit disk. They obtained that the isometric pointwise multipliers of these spaces are unimodular constants.

In this work we will study the properties of the symbols of isometric multiplication and weighted composition operators of BMOA space.

\section{Isometric multiplication operators}

The Hardy space $H^{2}(\mathbb{D})$ consists of all analytic functions on $\mathbb{D}$ whose power series have square summable coefficients. For every $f \in H^{2}$, the radial limit $f(\zeta)=\lim _{r \rightarrow 1^{-}} f(r \zeta)$ exists almost everywhere on $\partial \mathbb{D}$, and a standard norm on $H^{2}$ is given by

$$
\|f\|_{2}=\left(\int_{\partial \mathbb{D}}|f(\zeta)|^{2} d m(\zeta)\right)^{\frac{1}{2}}
$$

Here $d m$ is the Lebesgue measure on $\partial \mathbb{D}$ normalized so that $m(\partial \mathbb{D})=1$. Also, the norm on $H^{2}$ can be represented as

$$
\|f\|_{2}=\sup _{0<r<1}\left(\frac{1}{2 \pi} \int_{0}^{2 \pi}\left|f\left(r e^{i \theta}\right)\right|^{2} d \theta\right)^{\frac{1}{2}} .
$$

Let $X$ and $Y$ be metric spaces with metrics $d_{X}$ and $d_{Y}$. A map $T: X \rightarrow Y$ is called an isometry if for any $x_{1}, x_{2} \in X$ one has

$$
d_{Y}\left(T x_{1}, T x_{2}\right)=d_{X}\left(x_{1}, x_{2}\right) .
$$


Given two normed vector spaces $X$ and $Y$, a linear isometry is a linear map $T: X \rightarrow Y$ that preserves the norms,

$$
\|T x\|_{Y}=\|x\|_{X}
$$

for all $x \in X$.

If $M_{u}$ is an isometric operator of a Banach space $X \neq 0$, then it is also a bounded operator of $X$. Hence, if $M_{u}$ is an isometry of BMOA then $u$ must be an $H^{\infty}$ function with $H^{\infty}$-norm smaller than or equal to 1 . This can be seen in [18-20].

We will consider the question when the multiplication operator $M_{u}$ is an isometry of BMOA space. We start with the following proposition.

Proposition 1 If $M_{u}$ is an isometry of $B M O A$ space, then $u \in \mathrm{BMOA}$ and $\|u\|_{\mathrm{BMOA}}=1$.

Proof Suppose $M_{u}$ is an isometry of BMOA, then

$$
\left\|M_{u} f\right\|_{\mathrm{BMOA}}=\|u f\|_{\mathrm{BMOA}}=\|f\|_{\mathrm{BMOA}}
$$

for every $f \in$ BMOA. That is,

$$
|u(0) f(0)|+\sup _{a \in \mathbb{D}}\left\|u\left(\sigma_{a}\right) f\left(\sigma_{a}\right)-u(a) f(a)\right\|_{2}=|f(0)|+\sup _{a \in \mathbb{D}}\left\|f\left(\sigma_{a}\right)-f(a)\right\|_{2} .
$$

Letting $f=1$ in (1), we get

$$
\|u\|_{\mathrm{BMOA}}=|u(0)|+\sup _{a \in \mathbb{D}}\left\|u\left(\sigma_{a}\right)-u(a)\right\|_{2}=\|1\|_{\mathrm{BMOA}}=1 .
$$

It is easy to see that $\|u\|_{\mathrm{BMOA}}=1$ is a necessary but not sufficient condition for $M_{u}$ to be an isometry of BMOA space. The following is an example.

Example 1 Let $u=z$, then $\|z\|_{\mathrm{BMOA}}=1$, but $M_{z}$ is not an isometry of BMOA, since

$$
\left\|\sigma_{a}(z)\right\|_{\mathrm{BMOA}}=\left|\sigma_{a}(0)\right|+\sup _{b \in \mathbb{D}}\left\|\sigma_{a}\left(\sigma_{b}(z)\right)-\sigma_{a}(b)\right\|_{2}=1+|a|
$$

and

$$
\begin{aligned}
\left\|M_{z} \sigma_{a}(z)\right\|_{\mathrm{BMOA}} & =\left|0 \cdot \sigma_{a}(0)\right|+\sup _{b \in \mathbb{D}}\left\|\sigma_{b}(z) \sigma_{a}\left(\sigma_{b}(z)\right)-b \cdot \sigma_{a}(b)\right\|_{2} \\
& =\sup _{b \in \mathbb{D}} \sqrt{1-|b|^{2}\left|\sigma_{a}(b)\right|^{2}}=1
\end{aligned}
$$

for $a \neq 0$.

Now we describe the isometric multiplication operators of BMOA space in the following.

Proposition 2 Suppose $M_{u}$ is an isometry of BMOA, then the function $u$ has one of the following properties: 
(1) The function $u(z)$ is a constant function of modulus 1 ;

(2) There exists a real number $\lambda \in[0,1]$ such that $u(z)=\frac{c}{1-\lambda z}$. Here, $c=u(0)$, $\lambda=1-|u(0)|$.

Proof Suppose $M_{u}$ is an isometry of BMOA, let $f(z)=z$ in (1), then we can get

$$
\left\|M_{u} z\right\|_{\mathrm{BMOA}}=\|u z\|_{\mathrm{BMOA}}=\sup _{a \in \mathbb{D}}\left\|u\left(\sigma_{a}\right) \sigma_{a}-u(a) a\right\|_{2}=\|z\|_{\mathrm{BMOA}} .
$$

Moreover,

$$
\|z\|_{\mathrm{BMOA}}=\sup _{a \in \mathbb{D}}\left\|\sigma_{a}-a\right\|_{2}=\sup _{a \in \mathbb{D}} \sqrt{1-|a|^{2}}=1 .
$$

Hence,

$$
\sup _{a \in \mathbb{D}}\left\|u\left(\sigma_{a}\right) \sigma_{a}-u(a) a\right\|_{2}=1
$$

Let $f(z)=1+z$ in $(1)$, then

$$
\begin{aligned}
\|1+z\|_{\mathrm{BMOA}} & =1+\sup _{a \in \mathbb{D}}\left\|1+\sigma_{a}-(1+a)\right\|_{2} \\
& =1+\sup _{a \in \mathbb{D}}\left\|\sigma_{a}-a\right\|_{2}=1+1=2 .
\end{aligned}
$$

By (1) again, we obtain

$$
\begin{aligned}
& \left\|M_{u}(1+z)\right\|_{\mathrm{BMOA}} \\
& =\|u+u z\|_{\mathrm{BMOA}} \\
& =|u(0)|+\sup _{a \in \mathbb{D}}\left\|u\left(\sigma_{a}\right)+u\left(\sigma_{a}\right) \sigma_{a}-(u(a)+u(a) a)\right\|_{2} \\
& =|u(0)|+\sup _{a \in \mathbb{D}}\left\|u\left(\sigma_{a}\right)-u(a)+u\left(\sigma_{a}\right) \sigma_{a}-u(a) a\right\|_{2} .
\end{aligned}
$$

Since $\left\|M_{u}(1+z)\right\|_{\mathrm{BMOA}}=\|1+z\|_{\mathrm{BMOA}}$, we get

$$
|u(0)|+\sup _{a \in \mathbb{D}}\left\|u\left(\sigma_{a}\right)-u(a)+u\left(\sigma_{a}\right) \sigma_{a}-u(a) a\right\|_{2}=2
$$

For simplicity, we denote

$$
F_{a}(z)=u\left(\sigma_{a}(z)\right)-u(a), G_{a}(z)=u\left(\sigma_{a}(z)\right) \sigma_{a}(z)-u(a) a
$$

Now from (2)-(4), we have

$$
\sup _{a \in \mathbb{D}}\left\|F_{a}+G_{a}\right\|_{2}=2-|u(0)|=\sup _{a \in \mathbb{D}}\left\|F_{a}\right\|_{2}+\sup _{a \in \mathbb{D}}\left\|G_{a}\right\|_{2} .
$$


Due to the properties of supremum, triangle inequality of norm, and (5), we have

$$
\begin{aligned}
& \sup _{a \in \mathbb{D}}\left\|F_{a}\right\|_{2}^{2}+\sup _{a \in \mathbb{D}}\left\|G_{a}\right\|_{2}^{2}+2 \sup _{a \in \mathbb{D}}\left\|F_{a}\right\|_{2} \sup _{a \in \mathbb{D}}\left\|G_{a}\right\|_{2} \\
& \quad=\sup _{a \in \mathbb{D}}\left(\left\|F_{a}\right\|_{2}^{2}+\left\|G_{a}\right\|_{2}^{2}+\left\langle F_{a}, G_{a}\right\rangle+\left\langle G_{a}, F_{a}\right\rangle\right) \\
& \quad \leq \sup _{a \in \mathbb{D}}\left(\left\|F_{a}\right\|_{2}^{2}+\left\|G_{a}\right\|_{2}^{2}\right)+\sup _{a \in \mathbb{D}}\left(\left\langle F_{a}, G_{a}\right\rangle+\left\langle G_{a}, F_{a}\right\rangle\right) \\
& \quad \leq \sup _{a \in \mathbb{D}}\left\|F_{a}\right\|_{2}^{2}+\sup _{a \in \mathbb{D}}\left\|G_{a}\right\|_{2}^{2}+\sup _{a \in \mathbb{D}}\left(\left\langle F_{a}, G_{a}\right\rangle+\left\langle G_{a}, F_{a}\right\rangle\right) \\
& \quad \leq \sup _{a \in \mathbb{D}}\left\|F_{a}\right\|_{2}^{2}+\sup _{a \in \mathbb{D}}\left\|G_{a}\right\|_{2}^{2}+2 \sup _{a \in \mathbb{D}}\left\|F_{a}\right\|_{2}\left\|G_{a}\right\|_{2} \\
& \quad \leq \sup _{a \in \mathbb{D}}\left\|F_{a}\right\|_{2}^{2}+\sup _{a \in \mathbb{D}}\left\|G_{a}\right\|_{2}^{2}+2 \sup _{a \in \mathbb{D}}\left\|F_{a}\right\|_{2} \sup _{a \in \mathbb{D}}\left\|G_{a}\right\|_{2} .
\end{aligned}
$$

So we obtain

$$
\sup _{a \in \mathbb{D}}\left(\left\|F_{a}\right\|_{2}^{2}+\left\|G_{a}\right\|_{2}^{2}\right)=\sup _{a \in \mathbb{D}}\left\|F_{a}\right\|_{2}^{2}+\sup _{a \in \mathbb{D}}\left\|G_{a}\right\|_{2}^{2}
$$

and

$$
\begin{aligned}
\sup _{a \in \mathbb{D}}\left(\left\langle F_{a}, G_{a}\right\rangle+\left\langle G_{a}, F_{a}\right\rangle\right) & =2 \sup _{a \in \mathbb{D}}\left\|F_{a}\right\|_{2}\left\|G_{a}\right\|_{2} \\
& =2 \sup _{a \in \mathbb{D}}\left\|F_{a}\right\|_{2} \sup _{a \in \mathbb{D}}\left\|G_{a}\right\|_{2} .
\end{aligned}
$$

Consider two cases. In the first, there exists $a_{0} \in \mathbb{D}$ such that

$$
\left\langle F_{a_{0}}, G_{a_{0}}\right\rangle=\left\|F_{a_{0}}\right\|_{2}\left\|G_{a_{0}}\right\|_{2}=\sup _{a \in \mathbb{D}}\left\|F_{a}\right\|_{2} \sup _{a \in \mathbb{D}}\left\|G_{a}\right\|_{2}
$$

It follows that there exist $\lambda \geq 0$ and $a_{0} \in \mathbb{D}$ such that $F_{a_{0}}=\lambda G_{a_{0}}$. If $F_{a_{0}}=\lambda G_{a_{0}}$, we can obtain that $\lambda=1-|u(0)|$ from (2) and (3). There are two cases:

- If $\lambda=0$, then $u(z)$ is a constant function of modulus one;

- If $\lambda \neq 0$,then

$$
u\left(\sigma_{a_{0}}(z)\right)-u\left(a_{0}\right)=\lambda\left[u\left(\sigma_{a_{0}}(z)\right) \sigma_{a_{0}}(z)-u\left(a_{0}\right) a_{0}\right]
$$

for every $z \in \mathbb{D}$. Let $z=a_{0}$ in (7), then $\left(1-\lambda a_{0}\right) u\left(a_{0}\right)=u(0)$. It is easy to see that $u\left(a_{0}\right) \neq 0$.

- If $a_{0}=0$, we have $u(z)=\frac{u(0)}{1-\lambda z}=\frac{c}{1-\lambda z}$, where $c=u(0)$ is a constant;

- If $a_{0} \neq 0$, then $\lambda$ can be written as $\lambda=\frac{u\left(a_{0}\right)-u(0)}{u\left(a_{0}\right) a_{0}}$. Letting $z=\sigma_{a_{0}}(z)$ in (7), we obtain

$$
u(z)-u\left(a_{0}\right)=\lambda\left[u(z) z-u\left(a_{0}\right) a_{0}\right]
$$

that is,

$$
u(z)=u\left(a_{0}\right) \frac{1-\lambda a_{0}}{1-\lambda z}=\frac{u\left(a_{0}\right) u(0) a_{0}}{u\left(a_{0}\right) a_{0}-\left(u\left(a_{0}\right)-u(0)\right) z} .
$$


If $z=0$ in (8), then

$$
u(0)=u\left(a_{0}\right)\left(1-\lambda a_{0}\right) .
$$

This shows that $u(z)=\frac{u(0)}{1-\lambda z}$.

Now consider the second case and suppose that there exists a sequence $\left\{a_{n}\right\}$ in $\mathbb{D}$ converging to $\xi_{0} \in \partial \mathbb{D}$ such that

$$
\lim _{n \rightarrow \infty}\left\langle F_{a_{n}}, G_{a_{n}}\right\rangle=\lim _{n \rightarrow \infty}\left\|F_{a_{n}}\right\|_{2}\left\|G_{a_{n}}\right\|_{2}=1-|u(0)|
$$

Since

$$
\begin{aligned}
\left\langle F_{a}, G_{a}\right\rangle & =\left\langle u\left(\sigma_{a}\right)-u(a), u\left(\sigma_{a}\right) \sigma_{a}-u(a) a\right\rangle \\
& =\int_{\partial \mathbb{D}}\left|u\left(\sigma_{a}(\xi)\right)\right|^{2} \overline{\sigma_{a}(\xi)} d m(\xi)-|u(a)|^{2} \bar{a},
\end{aligned}
$$

we obtain

$$
\lim _{n \rightarrow \infty}\left\langle F_{a_{n}}, G_{a_{n}}\right\rangle=0=1-|u(0)|
$$

We get $|u(0)|=1$ from the above equation, so $u(z)$ is a constant function of modulus one.

It is a question whether $M_{u}$ is an isometry of BMOA space when $u$ has the form in Proposition 2. We have the following result.

Theorem 3 Let $u(z)=\frac{u(0)}{1-\lambda z}$, where $\lambda=1-|u(0)|$. Then $M_{u}$ is an isometry of BMOA space only if $|u(0)|=1$. Therefore, $M_{u}$ is an isometry of BMOA space if and only if $u(z)$ is a constant function of modulus 1 .

Proof Let $u(z)=\frac{u(0)}{1-\lambda z}$, then

$$
\left\|M_{u} 1\right\|_{\mathrm{BMOA}}=|u(0)|+\sup _{a \in \mathbb{D}}\left\|\frac{1}{1-\lambda \sigma_{a}(z)}-\frac{1}{1-\lambda a}\right\|_{2}=\|z\|_{\mathrm{BMOA}}=1 .
$$

By calculation, we have

$$
|u(0)|+\sup _{a \in \mathbb{D}}\left\|\frac{1}{1-\lambda \sigma_{a}(z)}-\frac{1}{1-\lambda a}\right\|_{2}=|u(0)|\left(1+\sqrt{\frac{\lambda^{2}}{1-\lambda^{2}}} \sup _{a \in \mathbb{D}} \sqrt{\frac{1-|a|^{2}}{|1-\lambda a|^{2}}}\right) .
$$

We want to get the extremum value of the function $f(a)=\frac{1-|a|^{2}}{|1-\lambda a|^{2}}$ for $a \in \mathbb{D}$. Suppose $a=$ $x+i y$, then

$$
f(a)=\frac{1-x^{2}-y^{2}}{(1-\lambda x)^{2}+(\lambda y)^{2}}
$$

Letting $f_{x}=0, f_{y}=0$, by calculation and analysis, we obtain that $x=\lambda, y=0$, which means that $a=\lambda$. Let $\lambda=1-|u(0)|$ in $|u(0)|\left(1+\frac{\lambda}{1-\lambda^{2}}\right)=1$. Through calculation, we can get $|u(0)|=$ 1 , which means $u(z)$ is a constant function of modulus 1 . 
Therefore, we get that $M_{u}$ is an isometry of BMOA space if and only if $u$ is a constant function of modulus 1 from Proposition 2.

\section{Isometric weighted composition operators}

In [14], the authors characterized the isometric composition operators of BMOA, they got the following results:

Lemma 4 ([14, Proposition 2.1]) A composition operator $C_{\varphi}$ is an isometry of BMOA if and only if $\varphi(0)=0$ and $\left\|C_{\varphi} f\right\|_{*}=\|f\|_{*}$ for all $f \in \mathrm{BMOA}$.

Lemma 5 ([14, Theorem 3.1]) The following conditions are equivalent:

- $\left\|C_{\varphi} f\right\|_{*}=\|f\|_{*}$ for all $f \in \mathrm{BMOA}$.

- $\left\|\sigma_{w} \circ \varphi\right\|_{*}=1$ for all $w \in \mathbb{D}$.

- The map $\varphi$ satisfies the following property: for every $w \in \mathbb{D}$, there is a sequence $\left(a_{n}\right)$ in $\mathbb{D}$ such that $\varphi\left(a_{n}\right) \rightarrow w$ and $\left\|\varphi_{a_{n}}\right\|_{2} \rightarrow 1$, as $n \rightarrow \infty$.

A weighted composition operator can be considered as a composition of multiplication and composition operators. We want to know the relationship between isometric weighted composition operator, composition operator, and multiplication operator.

In this section we will study the properties of isometric weighted composition operator of BMOA space. We assume that $\varphi(z)$ is an analytic self-map of $\mathbb{D}$ and continuous in $\overline{\mathbb{D}}$.

Lemma 6 If $M_{u, \varphi}$ is an isometry of $B M O A$ space, then $\|u\|_{\mathrm{BMOA}}=1$.

Proof If $M_{u, \varphi}$ is an isometry, then $\left\|M_{u, \varphi} f\right\|_{\mathrm{BMOA}}=\|f\|_{\mathrm{BMOA}}$ for every $f \in$ BMOA. Letting $f=1$ in $\left\|M_{u, \varphi} f\right\|_{\mathrm{BMOA}}=\|f\|_{\mathrm{BMOA}}$, we can get the result.

Proposition 7 If $M_{u, \varphi}$ is an isometry of BMOA space, then the functions $u(z)$ and $\varphi(z)$ have one of the following properties:

- The function $u(z)$ is a constant function of modulus 1 and $C_{\varphi}$ is an isometry of BMOA;

- There exist a real number $\lambda \in[0,1]$ and a constant $c$ such that $u(z)=\frac{c}{1-\lambda \varphi(z)}$, where $c=u(0)(1-\lambda \varphi(0))$ and $1-|u(0)|=\lambda(1-|u(0) \varphi(0)|)$.

Proof Suppose $M_{u, \varphi}$ is an isometry of BMOA space, then $\left\|M_{u, \varphi} f\right\|_{\mathrm{BMOA}}=\|f\|_{\mathrm{BMOA}}$ for every $f \in$ BMOA, that is,

$$
\begin{gathered}
|u(0) f(\varphi(0))|+\sup _{a \in \mathbb{D}}\left\|u\left(\sigma_{a}\right) f\left(\varphi\left(\sigma_{a}\right)\right)-u(a) f(\varphi(a))\right\|_{2} \\
=|f(0)|+\sup _{a \in \mathbb{D}}\left\|f\left(\sigma_{a}\right)-f(a)\right\|_{2} .
\end{gathered}
$$

Letting $f=1$ in (9), we get

$$
|u(0)|+\sup _{a \in \mathbb{D}}\left\|u\left(\sigma_{a}\right)-u(a)\right\|_{2}=1
$$

Letting $f=z$ in (9), we obtain

$$
|u(0) \varphi(0)|+\sup _{a \in \mathbb{D}}\left\|u\left(\sigma_{a}\right) \varphi\left(\sigma_{a}\right)-u(a) \varphi(a)\right\|_{2}=1
$$


Letting $f=1+z$ in (9) yields

$$
|u(0)+u(0) \varphi(0)|+\sup _{a \in \mathbb{D}}\left\|u\left(\sigma_{a}\right)-u(a)+u\left(\sigma_{a}\right) \varphi\left(\sigma_{a}\right)-u(a) \varphi(a)\right\|_{2}=2 .
$$

From (9)-(11), we have

$$
\begin{aligned}
& |u(0)+u(0) \varphi(0)|+\sup _{a \in \mathbb{D}}\left\|u\left(\sigma_{a}\right)-u(a)+u\left(\sigma_{a}\right) \varphi\left(\sigma_{a}\right)-u(a) \varphi(a)\right\|_{2} \\
& \quad=|u(0)|+|u(0) \varphi(0)|+\sup _{a \in \mathbb{D}}\left\|u\left(\sigma_{a}\right)-u(a)\right\|_{2}+\sup _{a \in \mathbb{D}}\left\|u\left(\sigma_{a}\right) \varphi\left(\sigma_{a}\right)-u(a) \varphi(a)\right\|_{2} .
\end{aligned}
$$

Due to the properties of supremum and triangle inequality, we can get

$$
|u(0)||1+\varphi(0)|=|u(0)|(1+|\varphi(0)|)
$$

and

$$
\begin{aligned}
\sup _{a \in \mathbb{D}}\left\|u\left(\sigma_{a}\right)-u(a)+u\left(\sigma_{a}\right) \varphi\left(\sigma_{a}\right)-u(a) \varphi(a)\right\|_{2} \\
=\sup _{a \in \mathbb{D}}\left(\left\|u\left(\sigma_{a}\right)-u(a)\right\|_{2}+\left\|u\left(\sigma_{a}\right) \varphi\left(\sigma_{a}\right)-u(a) \varphi(a)\right\|_{2}\right) \\
=\sup _{a \in \mathbb{D}}\left\|u\left(\sigma_{a}\right)-u(a)\right\|_{2}+\sup _{a \in \mathbb{D}}\left\|u\left(\sigma_{a}\right) \varphi\left(\sigma_{a}\right)-u(a) \varphi(a)\right\|_{2} .
\end{aligned}
$$

In the same way as in the proof of Proposition 2, we can get the existence of $0 \leq \lambda \leq 1$ and $a_{0} \in \mathbb{D}$ such that

$$
u\left(\sigma_{a_{0}}(z)\right)-u\left(a_{0}\right)=\lambda\left(u\left(\sigma_{a_{0}}(z)\right) \varphi\left(\sigma_{a_{0}}(z)\right)-u\left(a_{0}\right) \varphi\left(a_{0}\right)\right)
$$

or that of a sequence $\left\{a_{n}\right\}$ in $\mathbb{D}$ converging to $\xi_{0} \in \partial \mathbb{D}$ such that

$$
\begin{aligned}
& \lim _{n \rightarrow \infty}\left\langle u\left(\sigma_{a_{n}}\right)-u\left(a_{n}\right), u\left(\sigma_{a_{n}}\right) \varphi\left(\sigma_{a_{n}}\right)-u\left(a_{n}\right) \varphi\left(a_{n}\right)\right\rangle \\
& \quad=\lim _{n \rightarrow \infty}\left\|u\left(\sigma_{a_{n}}\right)-u\left(a_{n}\right)\right\|_{2}\left\|u\left(\sigma_{a_{n}}\right) \varphi\left(\sigma_{a_{n}}\right)-u\left(a_{n}\right) \varphi\left(a_{n}\right)\right\|_{2} \\
& \quad=(1-|u(0)|)(1-|u(0) \varphi(0)|) .
\end{aligned}
$$

First, we assume $u(0) \neq 0$. Letting $z=\sigma_{a_{0}}(z)$ in $u\left(\sigma_{a_{0}}(z)\right)-u\left(a_{0}\right)=\lambda\left(u\left(\sigma_{a_{0}}(z)\right) \varphi\left(\sigma_{a_{0}}(z)\right)-\right.$ $\left.u\left(a_{0}\right) \varphi\left(a_{0}\right)\right)$, we have

$$
u(z)(1-\lambda \varphi(z))=u\left(a_{0}\right)\left(1-\lambda \varphi\left(a_{0}\right)\right)
$$

- If $\lambda=0$, then $u(z)$ is a constant function of modulus one and $C_{\varphi}$ is an isometry on BMOA. The weighted composition operator $M_{u, \varphi}$ is constructed by an isometric multiplication operator and an isometric composition operator;

- If $\lambda \neq 0$, then $u(z)(1-\lambda \varphi(z))=u\left(a_{0}\right)\left(1-\lambda \varphi\left(a_{0}\right)\right)$, that is,

$$
u(z)=u\left(a_{0}\right) \frac{1-\lambda \varphi\left(a_{0}\right)}{1-\lambda \varphi(z)}
$$


Letting $z=0$, we have $u\left(a_{0}\right)\left(1-\lambda \varphi\left(a_{0}\right)\right)=u(0)(1-\lambda \varphi(0))$. That is,

$$
u(z)=\frac{c}{1-\lambda \varphi(z)}
$$

Here $c=u(0)(1-\lambda \varphi(0))$.

In the other hand, if

$$
\lim _{n \rightarrow \infty}\left\langle u\left(\sigma_{a_{n}}\right)-u\left(a_{n}\right), u\left(\sigma_{a_{n}}\right) \varphi\left(\sigma_{a_{n}}\right)-u\left(a_{n}\right) \varphi\left(a_{n}\right)\right\rangle=(1-|u(0)|)(1-|u(0) \varphi(0)|),
$$

then we can obtain that $|u(0)|=1$ or $|u(0) \varphi(0)|=1$.

- If $|u(0)|=1$, then $u(z)$ is a constant function of modulus one and the composition operator $C_{\varphi}$ is an isometry;

- If $|u(0)| \neq 1$ and $|u(0) \varphi(0)|=1$, we get a contradiction since $|u(0)|$ is less than 1 by (10) and $\varphi(z)$ is an analytic self-map of $\mathbb{D}$;

- If $|u(0)|=1$ and $|u(0) \varphi(0)|=1$, we get a contraction since $\varphi(z)$ is an analytic self-map of $\mathbb{D}$.

Secondly, let $u(0)=0$. Arguing as we did in the case $u(0) \neq 0$, we have:

- If $u\left(\sigma_{a_{0}}(z)\right)-u\left(a_{0}\right)=\lambda\left(u\left(\sigma_{a_{0}}(z)\right) \varphi\left(\sigma_{a_{0}}(z)\right)-u\left(a_{0}\right) \varphi\left(a_{0}\right)\right)$, then $u$ and $\varphi$ have properties similar to those in the case $u(0) \neq 0$;

- If $\lim _{n \rightarrow \infty}\left\langle u\left(\sigma_{a_{n}}\right)-u\left(a_{n}\right), u\left(\sigma_{a_{n}}\right) \varphi\left(\sigma_{a_{n}}\right)-u\left(a_{n}\right) \varphi\left(a_{n}\right)\right\rangle=(1-|u(0)|)(1-|u(0) \varphi(0)|)$, we get a contraction since the left-hand side of the equation is 0 , while the right-hand side is not.

In the following we consider whether $M_{u, \varphi}$ is an isometry of BMOA space when $u(z)$ has the form $\frac{c}{1-\lambda \varphi(z)}$.

Theorem 8 Let $u(z)=\frac{c}{1-\lambda \varphi(z)}$, where $c=u(0)(1-\lambda \varphi(0))$, then $M_{u, \varphi}$ is an isometry of $B M O A$ only if $u(z)$ is a constant function of modulus 1 . Therefore, $M_{u, \varphi}$ is an isometry of BMOA if and only if both $M_{u}$ and $C_{\varphi}$ are isometries of $B M O A$.

Proof If $u(z)=\frac{c}{1-\lambda \varphi(z)}$, let $f(z)=1-\lambda z$, then

$$
M_{u, \varphi} f(z)=c=u(0)(1-\lambda \varphi(0)) .
$$

Since $\left\|M_{u, \varphi} f\right\|_{\mathrm{BMOA}}=\|f\|_{\mathrm{BMOA}}$ and $\|1-\lambda z\|_{\mathrm{BMOA}}=1+\lambda$, we get

$$
|u(0)(1-\lambda \varphi(0))|=1+\lambda
$$

By calculation and analysis, we have $|u(0)|=1$, which means that $u(z)$ is a constant function of modulus 1.

Therefore, we obtain that if $M_{u, \varphi}$ is an isometry of BMOA, then $M_{u}$ is an isometry of BMOA. Then $M_{u, \varphi}$ is an isometry of BMOA if and only if both $M_{u}$ and $C_{\varphi}$ are isometries of BMOA. 


\section{Funding}

The author is partially supported by the National Natural Science Foundation of China $(11701056,11271388,11426046)$; Natural Science Foundation of Chongqing (cstc2018jcyjAx0595); Chongqing Key Laboratory of Social Economy and Applied Statistics.

\section{Competing interests}

The author declares that he has no competing interests.

Authors' contributions

Ligang Geng performed the research and wrote the paper. Author read and approved the final manuscript.

\section{Publisher's Note}

Springer Nature remains neutral with regard to jurisdictional claims in published maps and institutional affiliations.

Received: 24 November 2020 Accepted: 31 August 2021 Published online: 16 September 2021

\section{References}

1. Baernstein, A. II: Analytic functions of bounded mean oscillation. In: Aspects of Contemporary Complex Analysis (Proc. NATO Adv. Study Inst., Univ. Durham, Durham, 1979), pp. 3-36. Academic Press, London (1980)

2. Girela, D.: Analytic functions of bounded mean oscillation. In: Complex Function Spaces (Mekrijärvi, 1999), Univ. Joensuu Dept. Math. Rep. Ser., vol. 4, pp. 61-170. Univ. Joensuu, Joensuu (2001)

3. Garnett, J.B.: Bounded Analytic Functions. Academic Press, New York (1981)

4. Axler, S.: Multiplication operators on Bergman spaces. J. Reine Angew. Math. 1982(336), 19 (1982)

5. Feng, X., Zhang, K., Dong, J., Liu, X., Guan, C.: Multiplication operator with BMO symbols and Berezin transform. J. Funct. Spaces 2015, Article ID 754646 (2016)

6. Douglas, R.G., Sun, S., Zheng, D.: Multiplication operators on the Bergman space via analytic continuation. Adv. Math. 226(1), 541-583 (2011)

7. Guo, K., Huang, H.: Multiplication Operators on the Bergman Space. Lecture Notes in Mathematics Book Series (LNM), vol. 2145. Springer, Berlin (2015)

8. Forelli, F.: The isometries of $H^{P}$. Can. J. Math. 16, 721-728 (1964)

9. Cima, J., Wogen, W.: On isometries of Bloch spaces. III. J. Math. 24, 313-316 (1980)

10. de Leeuw, K., Rudin, W.: Extreme points and extremum problems in $H_{1}$. Pac. J. Math. 8, 467-485 (1958)

11. de Leeuw, K., Rudin, W., Wermer, J.: The isometries of some function spaces. Proc. Am. Math. Soc. 11, 694-698 (1960)

12. Hornor, W., Jamison, J.: Isometries of some Banach spaces of analytic functions. Integral Equ. Oper. Theory 41 410-425 (2001)

13. Novinger, W., Oberlin, D.: Linear isometries of some normed spaces of analytic functions. Can. J. Math. 37, 62-76 (1985)

14. Laitila, J.: Isometric composition operators on BMOA. Math. Nachr. 283(11), 1646-1653 (2010)

15. Le, T.: Normal and isometric weighted composition operators on the Fock space. Bull. Lond. Math. Soc. 46, 847-856 (2014)

16. Jaoua, N.: Isometric composition operators on the weighted Hardy spaces. Math. Nachr. 283(11), 1629-1636 (2010)

17. Panayappan, S.: Some isometric composition operators. Int. J. Contemp. Math. Sci. 5(13), 615-621 (2010)

18. Aleman, A., Duren, P., Martín, M.J., Vukotić, D.: Multiplicative isometries and isometric zero-divisors. Can. J. Math. 62(5), 961-974 (2010)

19. Girela, D., Merchán, N.: Multipliers and integration operators between conformally invariant spaces. Rev. R. Acad. Cienc. Exactas Fís. Nat., Ser. A Mat. 114(4), Article ID 181 (2020)

20. Vinogradov, S.A.: Multiplication and division in the space of analytic functions with area integrable derivative, and in some related spaces (in Russian). Zap. Nauč. Semin. POMI 222, 45-77 (1995), Issled. po Linein. Oper. i Theor. Funktsii 23, 45-77, 308; translation in J. Math. Sci. (New York) 87, no. 5 (1997), 3806-3827

\section{Submit your manuscript to a SpringerOpen ${ }^{\circ}$ journal and benefit from:}

- Convenient online submission

- Rigorous peer review

- Open access: articles freely available online

- High visibility within the field

Retaining the copyright to your article

Submit your next manuscript at $>$ springeropen.com 